\title{
The Epidemiological Characteristics of an Outbreak of 2019 Novel Coronavirus Diseases (COVID-19) - China, 2020
}

\author{
The Novel Coronavirus Pneumonia Emergency Response Epidemiology Team
}

\begin{abstract}
Background: An outbreak of 2019 novel coronavirus diseases (COVID-19) in Wuhan, Hubei Province, China has spread quickly nationwide. Here, we report results of a descriptive, exploratory analysis of all cases diagnosed as of February 11, 2020.

Methods: All COVID-19 cases reported through February 11, 2020 were extracted from China's Infectious Disease Information System. Analyses included the following: 1) summary of patient characteristics; 2) examination of age distributions and sex ratios; 3) calculation of case fatality and mortality rates; 4) geo-temporal analysis of viral spread; 5) epidemiological curve construction; and 6) subgroup analysis.

Results: A total of 72,314 patient records $-44,672$ $(61.8 \%)$ confirmed cases, $16,186(22.4 \%)$ suspected cases, 10,567 (14.6\%) clinically diagnosed cases (Hubei Province only), and 889 asymptomatic cases $(1.2 \%)$ - contributed data for the analysis. Among confirmed cases, most were aged $30-79$ years $(86.6 \%)$, diagnosed in Hubei (74.7\%), and considered mild $(80.9 \%)$. A total of 1,023 deaths occurred among confirmed cases for an overall case fatality rate of $2.3 \%$. The COVID-19 spread outward from Hubei Province sometime after December 2019, and by February 11, $2020,1,386$ counties across all 31 provinces were affected. The epidemic curve of onset of symptoms peaked around January 23-26, then began to decline leading up to February 11. A total of 1,716 health workers have become infected and 5 have died $(0.3 \%)$.

Conclusions: COVID-19 epidemic has spread very quickly taking only 30 days to expand from Hubei to the rest of Mainland China. With many people returning from a long holiday, China needs to prepare for the possible rebound of the epidemic.
\end{abstract}

\section{Introduction}

A cluster of pneumonia cases of unknown origin in Wuhan, China caused concern among health officials in late December 2019. On December 31, an alert was issued by the Wuhan Municipal Health Commission, a rapid response team was sent to Wuhan by the Chinese Center for Disease Control and Prevention (China CDC), and a notification was made to the World Health Organization (WHO) (1-4). Likely potential causes including influenza, avian influenza, adenovirus, severe acute respiratory syndrome coronavirus (SARS-CoV), and Middle East respiratory syndrome coronavirus (MERS-CoV) were ruled out. Epidemiological investigation implicated Wuhan's Huanan Seafood Wholesale Market, which was shut down and disinfected, and active case finding was initiated and vigorously pursued (2,4-5).

On January 7, 2020, the causative pathogen was identified as a novel coronavirus, and genomic characterization and test method development ensued (2-6). Now named 2019-nCoV, the virus is distinct from both SARS-CoV and MERS-CoV, yet closely related (5,7). Early cases suggested that COVID-19 (i.e. the new name for disease caused by the novel coronavirus) may be less severe than SARS and MERS. However, illness onset among rapidly increasing numbers of people and mounting evidence of humanto-human transmission suggests that $2019-\mathrm{nCoV}$ is more contagious than both SARS-CoV and MERS$\mathrm{CoV}(3,8-11)$.

On January 20, China's “National Infectious Diseases Law" was amended to make 2019-novel coronavirus diseases (COVID-19) a Class B notifiable disease and its "Frontier Health and Quarantine Law" was amended to support the COVID-19 outbreak response effort. Then, on January 23, the Chinese Government began to limit movement of people in and out of Wuhan, and two days later, it announced its highest-level commitment and mobilized all sectors to respond to the epidemic and prevent further spread of COVID19. Characterization of the epidemiological features of COVID-19 is crucial for the development and implementation of effective control strategies. Here, we report the results of a descriptive, exploratory analysis of all cases found through February 11, 2020. 


\section{Method}

\section{Study Design}

This study was a descriptive, exploratory analysis of all cases of COVID-19 diagnosed nationwide in China as of the end of February 11, 2020. As such, it in some respects uses a cross-sectional study design and hence, we have used the STROBE Guidelines (www.equatornetwork.org) to aid our thorough reporting of this observational study.

A public health emergency was declared, and a formal investigation began on December 31, 2019, supported by city (Wuhan Municipal Health Commission and Wuhan CDC), provincial (Health Commission of Hubei Province and Hubei Provincial CDC), and national (National Health Commission and China CDC) authorities and resources. This study was reviewed by the China CDC Institutional Review Board via a fast-track mechanism. Although individual informed consent was not required for this study, all data were handled as a deidentified set to protect patient privacy and confidentiality.

\section{Data Source}

By categorizing COVID-19 as a Class B notifiable disease, Chinese law required all cases to be immediately reported to China's Infectious Disease Information System. Entry of each case into the system was performed by local epidemiologists and public health workers who investigated and collected information on possible exposures. All case records contain national identification numbers, and therefore, all cases have records in the system and no records are duplicated. All data contained in all COVID-19 case records in the Infectious Disease Information System through the end of February 11, 2020 were extracted from the system as a single dataset and were then stripped of all personal identifying information. No sampling was done to achieve a predetermined study size and no eligibility criteria were used-all cases were included.

\section{Variables}

Patient characteristics were collected at baseline, meaning the time of diagnosis, epidemiological investigation, and entry into the Infectious Disease Information System. Patients were categorized as health workers for the occupation variable if they had active employment of any kind in a health facility (i.e. this category did not just include physicians and nurses). Patients were categorized as having a Wuhanrelated exposure if they had recently resided in or visited Wuhan or if they had close contact with someone who had. The comorbid conditions variable was determined upon epidemiological investigation by patient self-reported medical history, which was not independently verified using medical records for all cases. The severity of symptoms variable was categorized as mild, severe, or critical. Mild included non-pneumonia and mild pneumonia cases. Severe was characterized by dyspnea, respiratory frequency $\geq$ $30 /$ minute, blood oxygen saturation $\leq 93 \%, \mathrm{PaO}_{2} / \mathrm{FiO}_{2}$ ratio $<300$, and/or lung infiltrates $>50 \%$ within $24-48$ hours. Critical cases were those that exhibited respiratory failure, septic shock, and/or multiple organ dysfunction/failure.

As some variables of interest (i.e., Wuhan-related exposure, comorbid condition, and case severity) are not required fields when creating records in the Infectious Disease Information System, some records have missing data for these variables.

For construction of epidemiological curves, date of onset was defined as the date on which patients selfreported the start of either fever or cough during epidemiological investigation. Cases were categorized as confirmed, suspected, clinically diagnosed (Hubei Province only), or asymptomatic. Confirmed cases were diagnosed based on positive viral nucleic acid test results on throat swab samples (some samples were tested retrospectively). Suspected cases were diagnosed clinically based on symptoms and exposures. Clinically diagnosed cases were suspected cases with lung imaging features consistent with coronavirus pneumonia. Asymptomatic cases were diagnosed based on positive viral nucleic acid test results but without any COVID19 symptoms (e.g., fever, dry cough). The date of positive viral nucleic acid test result is used as onset date for asymptomatic cases

\section{Analysis}

For confirmed cases, demographic and clinical characteristics were summarized using descriptive statistics. Age distribution graphs were constructed using patient age at baseline for confirmed cases diagnosed in Wuhan, Hubei Province (including Wuhan), and China (including Hubei Province). Sex ratio (i.e., male:female [M:F] ratio) was also calculated. Case fatality rates were calculated as the total number of deaths (numerator) divided by the total number of cases (denominator), expressed as a percent. Observed time was summarized using person-days (PD) and 
mortality was calculated as the number of deaths (numerator) divided by the total observed time (denominator), expressed per 10 PD.

For geo-temporal analysis, the county-level location of each case at time of diagnosis was used to build color-coded maps of China to indicate the numbers of cases in each province on December 31, 2019; January 10, 2020; January 31, 2020; and February 11, 2020. This analysis was performed using ArcGIS Desktop software (version 10.6; Esri; Redlands, California, USA).

The epidemiological curve for all cases was constructed by plotting the number of cases (y-axis) versus self-reported date of symptom onset (x-axis). Date of symptom onset for confirmed, suspected, clinically diagnosed, and asymptomatic cases were stacked to show total cases over time. The epidemiological curve for confirmed cases was also overlaid with the number of cases versus date of diagnosis to show the delay between onset of symptoms and diagnosis of disease.

Two subgroups were also analyzed separately using epidemiological curves: confirmed cases diagnosed outside of Hubei Province (with and without Wuhan- related exposure) and all cases diagnosed among health workers (confirmed, suspected, clinically diagnosed, and asymptomatic).

\section{Results}

\section{Patients}

A total of 72,314 unique records were extracted and data from all records were included in the analysis. Thus, all 72,314 individuals diagnosed with COVID19 as of February 11, 2020, were included in the analysis. Among them, 44,672 cases (61.8\%) were confirmed, 16,186 cases $(22.4 \%)$ were suspected, 10,567 cases $(14.6 \%)$ were clinically diagnosed, and 889 cases $(1.2 \%)$ were asymptomatic.

Baseline characteristics of confirmed cases $(\mathrm{n}=44,672)$ are presented in Table 1 . A majority were aged 30-69 years $(77.8 \%)$, male $(51.4 \%)$, farmers or laborers $(22.0 \%)$, and diagnosed in Hubei Province $(74.7 \%)$. Most patients reported Wuhan-related exposures $(85.8 \%)$ and were classified as mild cases $(80.9 \%)$.

TABLE 1. Patients, deaths, and case fatality rates, as well as observed time and mortality for $n=44,672$ confirmed COVID-19 cases in Mainland China as of February 11, 2020.

\begin{tabular}{|c|c|c|c|c|c|}
\hline Baseline characteristics & $\begin{array}{c}\text { Confirmed cases, } \\
\mathrm{N}(\%) \\
\end{array}$ & $\begin{array}{c}\text { Deaths, } \\
\mathbf{N}(\%)\end{array}$ & $\begin{array}{l}\text { Case fatality } \\
\text { rate, } \%\end{array}$ & $\begin{array}{c}\text { Observed time, } \\
\text { PD }\end{array}$ & $\begin{array}{l}\text { Mortality, } \\
\text { per } 10 \mathrm{PD}\end{array}$ \\
\hline Overall & 44,672 & 1,023 & 2.3 & 661,609 & 0.015 \\
\hline \multicolumn{6}{|l|}{ Age, years } \\
\hline $0-9$ & $416(0.9)$ & - & - & 4,383 & - \\
\hline $10-19$ & $549(1.2)$ & $1(0.1)$ & 0.2 & 6,625 & 0.002 \\
\hline $20-29$ & $3,619(8.1)$ & $7(0.7)$ & 0.2 & 53,953 & 0.001 \\
\hline $30-39$ & $7,600(17.0)$ & $18(1.8)$ & 0.2 & 114,550 & 0.002 \\
\hline $40-49$ & $8,571(19.2)$ & $38(3.7)$ & 0.4 & 128,448 & 0.003 \\
\hline $50-59$ & $10,008(22.4)$ & $130(12.7)$ & 1.3 & 151,059 & 0.009 \\
\hline $60-69$ & $8,583(19.2)$ & $309(30.2)$ & 3.6 & 128,088 & 0.024 \\
\hline $70-79$ & $3,918(8.8)$ & $312(30.5)$ & 8.0 & 55,832 & 0.056 \\
\hline$\geq 80$ & $1,408(3.2)$ & $208(20.3)$ & 14.8 & 18,671 & 0.111 \\
\hline \multicolumn{6}{|l|}{ Sex } \\
\hline Male & $22,981(51.4)$ & $653(63.8)$ & 2.8 & 342,063 & 0.019 \\
\hline Female & $21,691(48.6)$ & $370(36.2)$ & 1.7 & 319,546 & 0.012 \\
\hline \multicolumn{6}{|l|}{ Occupation } \\
\hline Service industry & $3,449(7.7)$ & $23(2.2)$ & 0.7 & 54,484 & 0.004 \\
\hline Farmer/laborer & $9,811(22.0)$ & $139(13.6)$ & 1.4 & 137,992 & 0.010 \\
\hline Health worker & $1,716(3.8)$ & $5(0.5)$ & 0.3 & 28,069 & 0.002 \\
\hline Retiree & $9,193(20.6)$ & $472(46.1)$ & 5.1 & 137,118 & 0.034 \\
\hline Other/none & $20,503(45.9)$ & $384(37.5)$ & 1.9 & 303,946 & 0.013 \\
\hline
\end{tabular}


TABLE 1. (continued)

\begin{tabular}{|c|c|c|c|c|c|}
\hline Baseline characteristics & $\begin{array}{c}\text { Confirmed cases, } \\
\mathrm{N}(\%) \\
\end{array}$ & $\begin{array}{c}\text { Deaths, } \\
\mathbf{N}(\%) \\
\end{array}$ & $\begin{array}{c}\text { Case fatality } \\
\text { rate, } \%\end{array}$ & $\begin{array}{c}\text { Observed time, } \\
\text { PD }\end{array}$ & $\begin{array}{l}\text { Mortality, } \\
\text { per } 10 \text { PD }\end{array}$ \\
\hline \multicolumn{6}{|l|}{ Province } \\
\hline Hubei & $33,367(74.7)$ & $979(95.7)$ & 2.9 & 496,523 & 0.020 \\
\hline Other & $11,305(25.3)$ & $44(4.3)$ & 0.4 & 165,086 & 0.003 \\
\hline \multicolumn{6}{|l|}{ Wuhan-related exposure* } \\
\hline Yes & $31,974(85.8)$ & $853(92.8)$ & 2.7 & 486,612 & 0.018 \\
\hline No & $5,295(14.2)$ & $66(7.2)$ & 1.2 & 71,201 & 0.009 \\
\hline Missing & 7,403 & 104 & 2.8 & 103,796 & 0.010 \\
\hline \multicolumn{6}{|l|}{ Comorbid condition $^{\dagger}$} \\
\hline Hypertension & $2,683(12.8)$ & $161(39.7)$ & 6.0 & 42,603 & 0.038 \\
\hline Diabetes & $1,102(5.3)$ & $80(19.7)$ & 7.3 & 17,940 & 0.045 \\
\hline Cardiovascular disease & $873(4.2)$ & $92(22.7)$ & 10.5 & 13,533 & 0.068 \\
\hline Chronic respiratory disease & $511(2.4)$ & $32(7.9)$ & 6.3 & 8,083 & 0.040 \\
\hline Cancer (any) & $107(0.5)$ & $6(1.5)$ & 5.6 & 1,690 & 0.036 \\
\hline None & $15,536(74.0)$ & $133(32.8)$ & 0.9 & 242,948 & 0.005 \\
\hline Missing & $23,690(53.0)$ & $617(60.3)$ & 2.6 & 331,843 & 0.019 \\
\hline \multicolumn{6}{|l|}{ Case severity ${ }^{\S}$} \\
\hline Mild & $36,160(80.9)$ & - & - & - & - \\
\hline Severe & $6,168(13.8)$ & - & - & - & - \\
\hline Critical & $2,087(4.7)$ & $1,023(100)$ & 49.0 & 31,456 & 0.325 \\
\hline Missing & $257(0.6)$ & - & - & - & - \\
\hline \multicolumn{6}{|l|}{ Period (by date of onset) } \\
\hline Before Dec 31, 2019 & $104(0.2)$ & $15(1.5)$ & 14.4 & 5,142 & 0.029 \\
\hline Jan $1-10,2020$ & $653(1.5)$ & $102(10.0)$ & 15.6 & 21,687 & 0.047 \\
\hline Jan 11-20, 2020 & $5,417(12.1)$ & $310(30.3)$ & 5.7 & 130,972 & 0.024 \\
\hline Jan 21-31, 2020 & $26,468(59.2)$ & $494(48.3)$ & 1.9 & 416,009 & 0.012 \\
\hline After Feb 1, 2020 & $12,030(26.9)$ & $102(10.0)$ & 0.8 & 87,799 & 0.012 \\
\hline
\end{tabular}

Abreviation: PD, person-days. -, not applicable.

* The Wuhan-related exposure variable, only includes a total of 37,269 patients and 919 deaths and these values were used to calculate percentages in the confirmed cases and deaths columns.

$\dagger$ The comorbid condition variable, only includes a total of 20,812 patients and 504 deaths and these values were used to calculate percentages in the confirmed cases and deaths columns.

$\S$ The case severity variable, only includes a total of 44,415 patients and 1,023 deaths and these values were used to calculate percentages in the confirmed cases and deaths columns.

\section{Deaths, Case Fatality Rates, and Mortality}

As shown in Table 1 , a total of 1,023 deaths have occurred among 44,672 confirmed cases for an overall case fatality rate of $2.3 \%$. Additionally, these 1,023 deaths occurred during 661,609 PD of observed time, for a mortality rate of $0.015 / 10 \mathrm{PD}$.

The $\geq 80$ age group had the highest case fatality rate of all age groups at $14.8 \%$. Case fatality rate for males was $2.8 \%$ and for females was $1.7 \%$. By occupation, patients who reported being retirees had the highest case fatality rate at $5.1 \%$, and patients in Hubei Province had a $>7$-fold higher case fatality rate at $2.9 \%$ compared to patients in other provinces $(0.4 \%)$. While patients who reported no comorbid conditions had a case fatality rate of $0.9 \%$, patients with comorbid conditions had much higher rates- $10.5 \%$ for those with cardiovascular disease, $7.3 \%$ for diabetes, $6.3 \%$ for chronic respiratory disease, $6.0 \%$ for hypertension, and $5.6 \%$ for cancer. Case fatality rate was also very high for cases categorized as critical at $49.0 \%$.

\section{Age Distribution and Sex Ratio}

The age distribution of cases in Wuhan only, in Hubei Province overall, and in China overall are 
presented in Figure 1. The proportion of confirmed cases 30-79 years of age at baseline (i.e., date of diagnosis) was $89.8 \%$ for cases in Wuhan city versus $88.6 \%$ in Hubei overall (which includes Wuhan) and $86.6 \%$ in China overall (which includes Hubei Province and all 30 other provincial-level administrative divisions, or PLADs). The male-tofemale ratio was 0.99:1 in Wuhan, 1.04:1 in Hubei, and 1.06:1 in China overall.

\section{Geo-Temporal Findings}

On January 19, 2020, National Health Commission of the People's Republic of China confirmed that Guangdong Provincial CDC reported first imported cases of COVID-19, via the Chinese Infectious Diseases Reporting System. This was the first time COVID-19 had been reported outside of Hubei Province via the System. As of January 22, 2020, a total of 301 confirmed COVID-19 cases were reported from 83 counties in 23 provinces. On January 30, 2020, Xizang Autonomous Region (Tibet) reported its first confirmed COVID-19 case coming from Hubei Province. Thus, COVID-19 cases have been reported from all 31 PLADs (Figure 2).

As of February 11, 2020, a total 44,672 confirmed cases were reported from 1,386 counties of 31 provinces, autonomous regions, and municipalities and Hubei Province accounted for $74.7 \%$ (Figure 2E). Among them, $0.2 \%$ of cases had onset of illness before December 31, 2019 and all were from Hubei Province (Figure 2A); $1.7 \%$ had onset of illness during January $1-10,2020$, distributed in 113 counties of 22 PLADs and Hubei Province accounted for $88.5 \%$ (Figure 2B); $13.8 \%$ had onset of illness during January 11-20, 2020 , distributed in 627 counties of 30 PLADs and Hubei Province accounted for $77.6 \%$ (Figure 2C); $73.1 \%$ had onset of illness during January 21-31, 2020, distributed in 1310 counties of 31 PLADs and Hubei Province accounted for $74.7 \%$ (Figure 2D).

\section{Epidemiological Curve}

Figure 3A shows the COVID-19 epidemic curve with number of cases plotted by date of patient onset of symptoms from December 8, 2019 to February 11, 2020. Confirmed, suspected, clinically diagnosed, and asymptomatic cases are stacked to show total daily cases by date of symptom onset. The inset shows that in December 2019 only 0-22 cases/day began to experience symptoms. The peak onset of symptoms for all cases overall occurred on February 1, 2020. Since then, onset of illness has declined.

Figure 3B shows the same COVID-19 epidemic curve for confirmed cases only with number of cases plotted by date of patients' onset of symptoms from December 8, 2019 to February 11, 2020. These data are overlaid with confirmed cases plotted by date of diagnosis to show the lag between the time patients fall ill and the time they actually are diagnosed and are reported to the Infectious Disease Information System. Although for confirmed cases onset of illness peaked around January 23-27, diagnosis of infection by nucleic acid testing of throat swabs did not peak until February 4 .

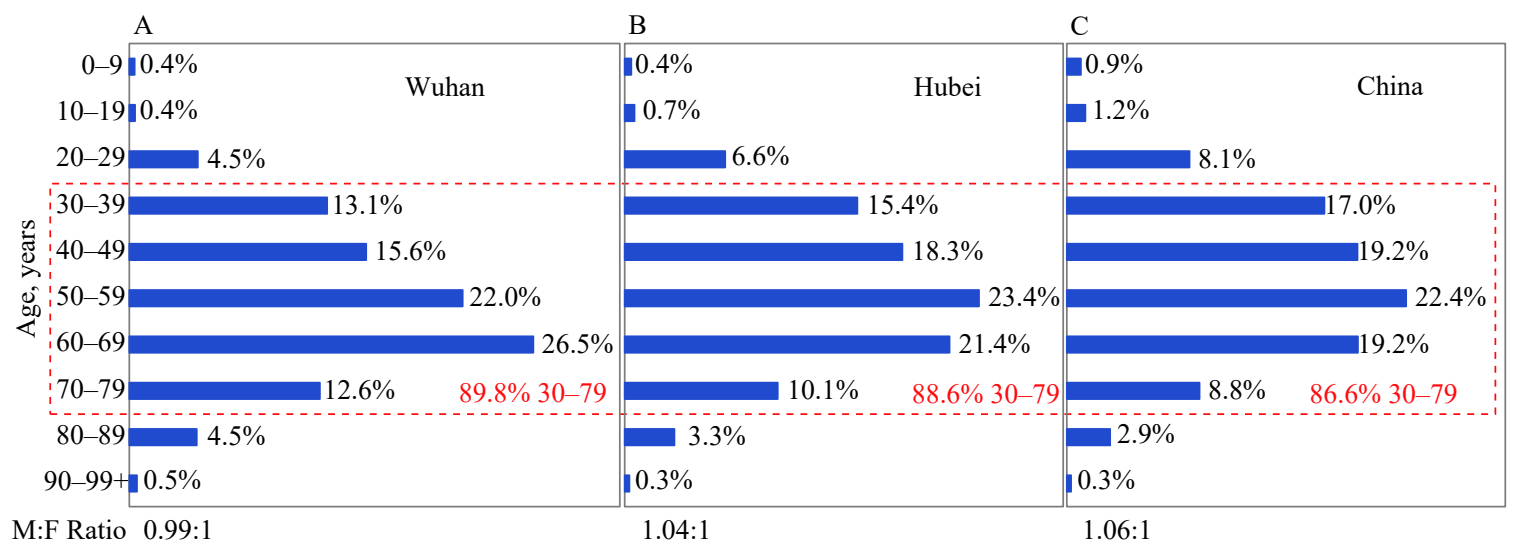

FIGURE 1. Age distribution and sex ratio of all confirmed COVID-19 cases in China through February 11, 2020. (A) patients diagnosed in the city of Wuhan only; (B) patients diagnosed in Hubei Province, which includes Wuhan as its capital city; and (C) patients diagnosed in China overall, including Hubei Province and all 30 other provincial-level administrative divisions (PLADs). Dashed red line highlights the proportion of patients in the 30-79 years age range. Sex ratio (i.e. male-to-female [M:F] ratio) is shown below each graph. 


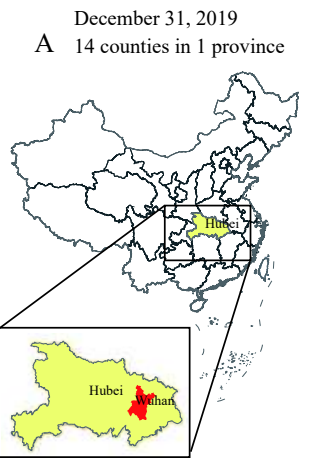

January 10,2020
B 113 counties in 20 provinces

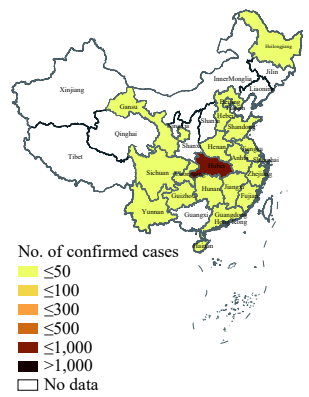

January 20, 2020

C 627 counties in 30 provinces

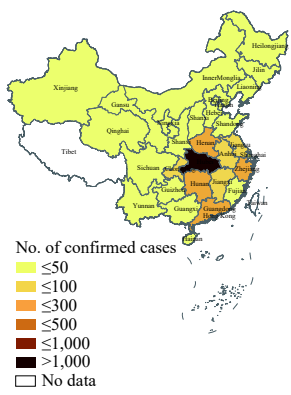

January 31,2020

D 1,310 counties in 31 provinces E 1,386 counties in 31 provinces

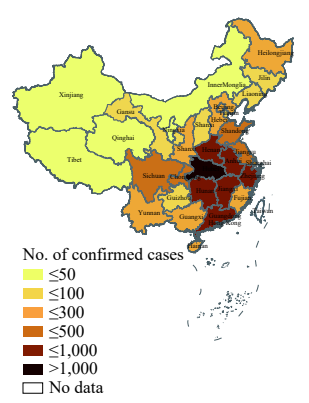

February 11, 2020

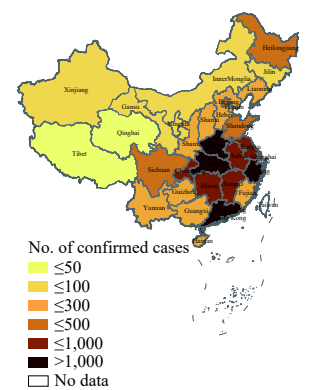

FIGURE 2. Geo-temporal spread of COVID-19 in China through February 11, 2020. (A) a total of 14 county-level administrative areas (hereafter counties) in Hubei Province only (inset) had reported cases as of December 31, 2019; (B) by January 10, 2020, 113 counties in 20 PLADs had reported cases with the highest prevalence still in Hubei Province; (C) nine days later, on January 20,627 counties in 30 PLADs had reported cases and PLADs neighboring Hubei Province observed increasing prevalence; (D) by the end of January 31, 1,310 counties across all 31 PLADs were affected and prevalence in the central, south, and south-central regions had risen dramatically; $(E)$ by the end of February 11, 1,386 counties nationwide were affected and prevalence in the south-central PLADs had risen to the level of Hubei.

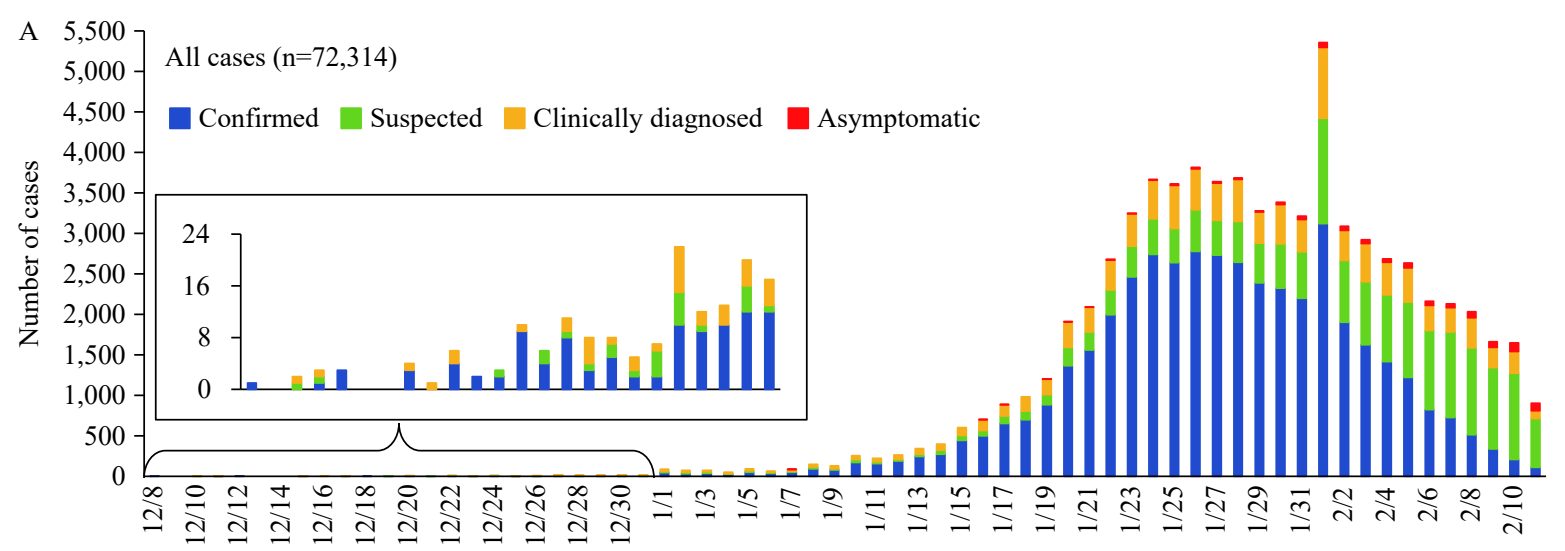

Date of symptom onset

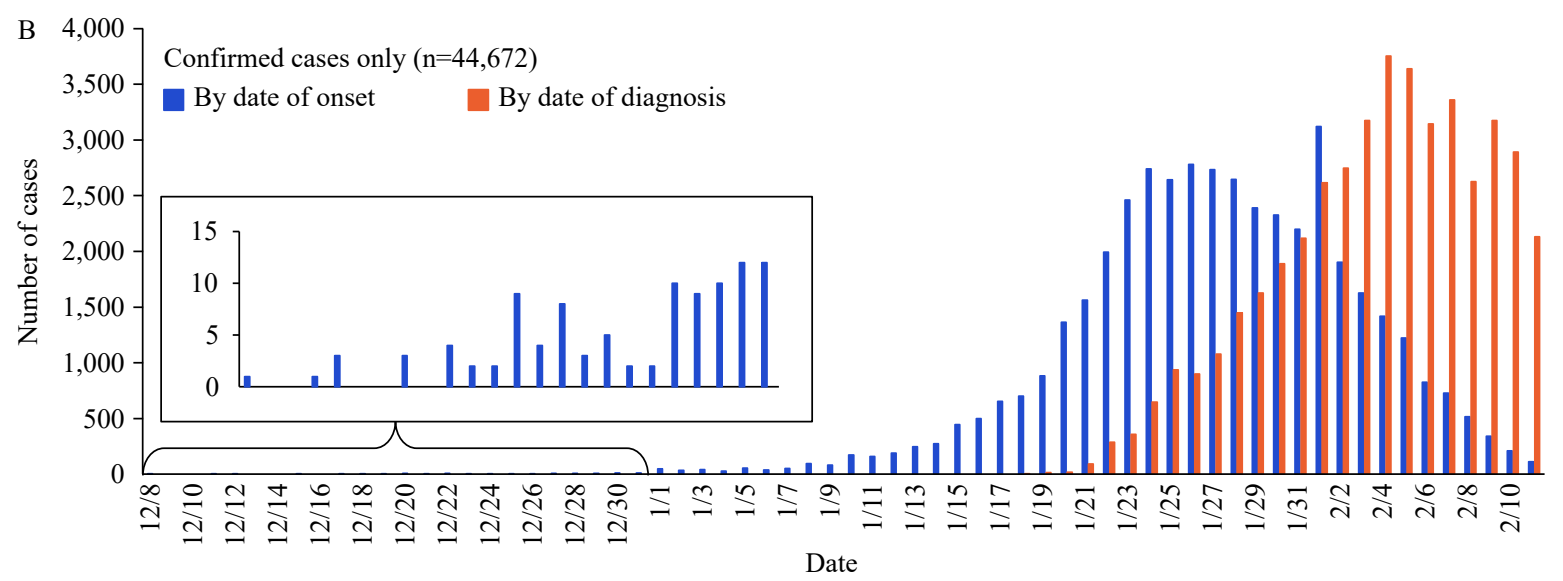

FIGURE 3. Epidemiological curves of COVID-19 in China through February 11, 2020. (A) the epidemiological curve shows the progression of illness in the outbreak over time from December 8, 2019 to February 11, 2020. A total of 72,314 cases are shown and confirmed cases (blue) are compared to suspected cases (green), clinically diagnosed cases (yellow), and asymptomatic cases (red). The inset shows a zoomed-in view of all days in December, when total daily count remained below 24 cases; (B) the epidemiological curve shows the progression of illness in the outbreak over time from December 8 , 2019 to February 11, 2020 for confirmed cases only (blue). The number of cases diagnosed each day is also shown for confirmed cases only (orange). The inset shows a zoomed-in view of all days in December, when total daily count remained below 15 cases. 


\section{Subgroup Findings}

Figure 4 shows the COVID-19 epidemic curve with the number of cases plotted by date of onset of symptoms from December 18, 2019 to February 11, 2020 for two subgroups-confirmed cases found outside of Hubei Province (Figure 4A) and all cases among health workers nationwide (Figure 4B). Peak timing of onset of symptoms among cases outside of Hubei Province occurred on January 27. Most of these cases $(85.8 \%)$ reported having recently resided in or visited Wuhan or having had close contact with an infected individual from Wuhan. Peak timing of onset of symptoms among health worker cases occurred on February 1. In the 422 medical facilities serving COVID-19 patients, a total of 3,019 health workers have been infected (1,716 confirmed cases), and 5 have died.

Confirmed cases, case severity, and case fatality rates among health workers in different areas of China and different time periods are presented in Table 2 . A total of 1,080 confirmed cases among health workers have been found in Wuhan, accounting for $64.0 \%$ of

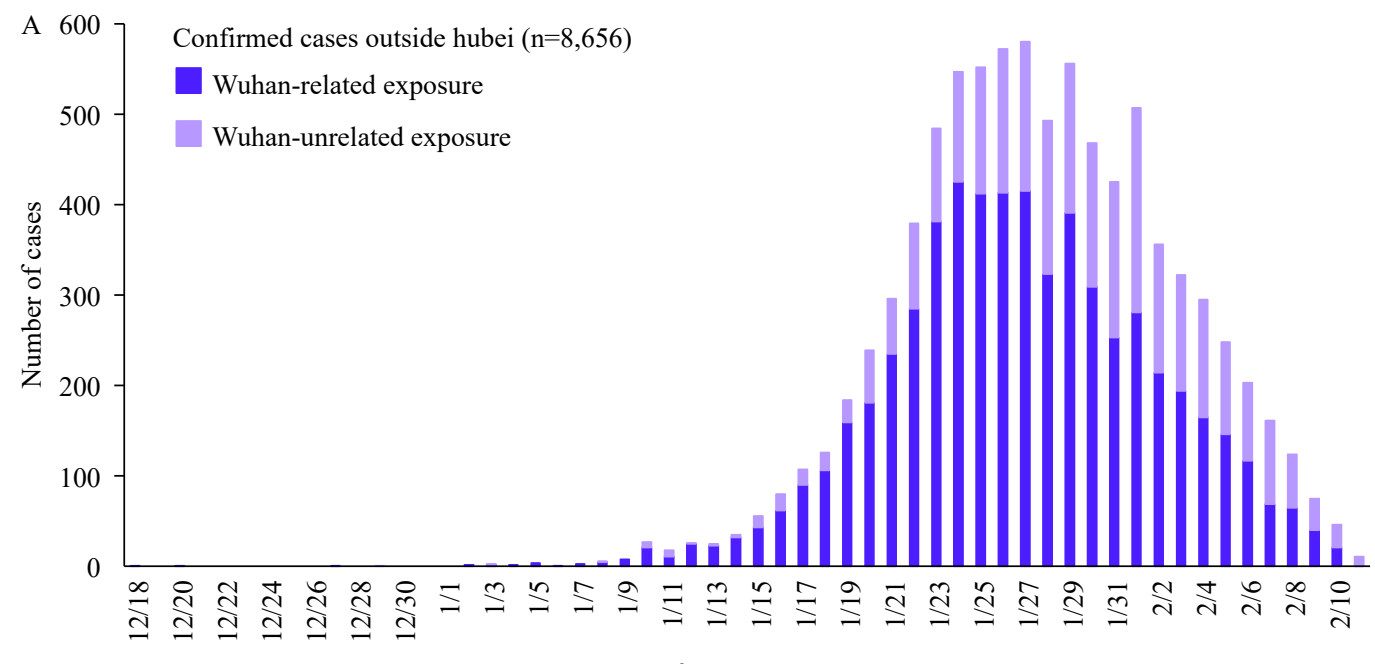

Date of symptom onset

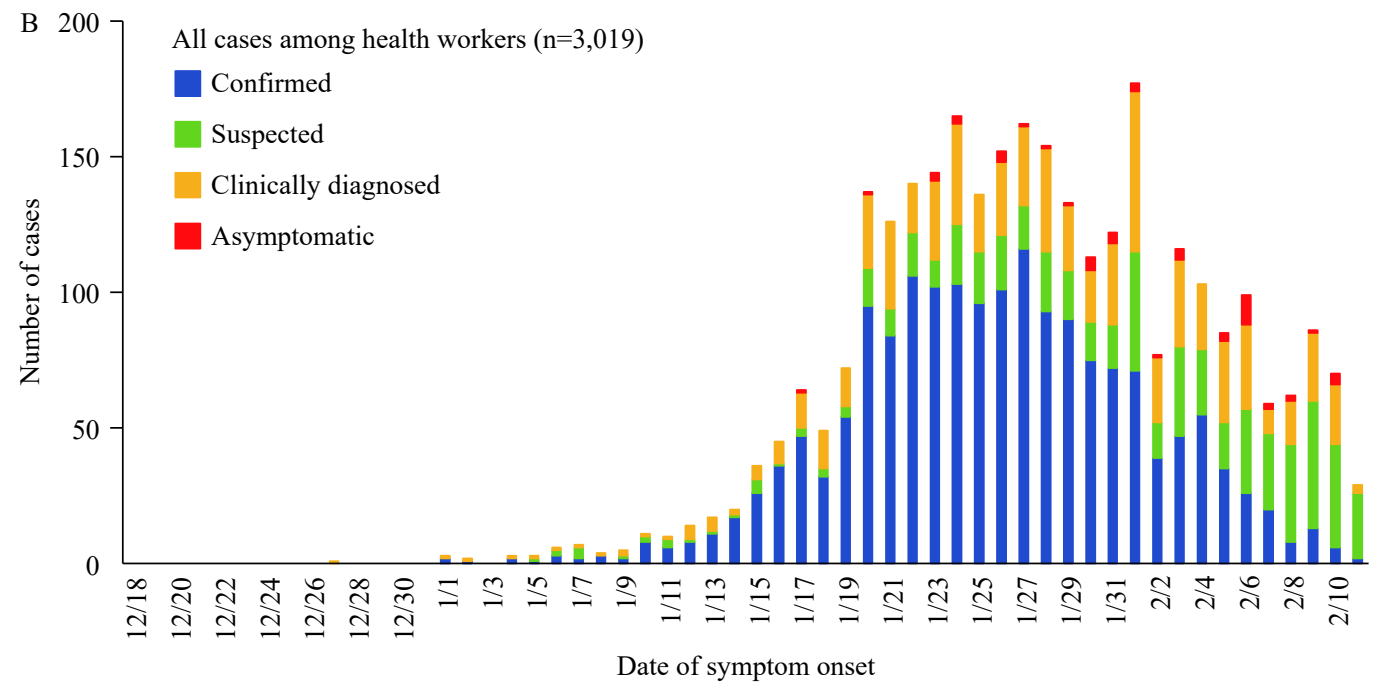

FIGURE 4. Subgroup epidemiological curves of COVID-19 in China through February 11, 2020. (A) subgroup analysis of confirmed cases discovered outside of Hubei Province only. The epidemiological curve shows the progression of illness in the outbreak over time from the onset of symptoms of the first case outside Hubei Province on December 18, 2019 through the end of February 11, 2020. Total confirmed cases outside Hubei Province, and Wuhan-related exposure (dark purple) versus Wuhan-unrelated exposure (light purple), are shown; (B) subgroup analysis of all cases among health workers only. The epidemiological curve shows the progression of illness in the outbreak over time from the onset of symptoms of the first health worker case on December 27, 2019 through the end of February 11, 2020. Total cases among health workers and confirmed (blue) versus suspected (green), clinically diagnosed (yellow), and asymptomatic (red) cases are shown. 
national total. An additional 394 health worker cases $(23.3 \%)$ were found in Hubei Province (excluding Wuhan), and 214 cases (12.7\%) were found in the other 30 PLADs. The proportion of health worker cases that were severe or critical was $17.7 \%$ in Wuhan, $10.4 \%$ in Hubei Province, $7.0 \%$ in the remaining 30 PLADs, and $14.6 \%$ overall. The proportion of health worker cases in Wuhan classified as severe or critical declined from $38.9 \%$ in early January to $12.7 \%$ in early February. In China overall, the severe or critical cases among health workers also declined-from $45.0 \%$ in early January to $8.7 \%$ in early February.

\section{Discussion}

A main finding of this characterization and exploratory analysis of the first 72,314 cases of COVID-19 found in China in the 40 days between first recognition of the outbreak of pneumonia with unknown etiology on December 31, 2019 to the end of the study period on February 11, 2020 is that this novel coronavirus is highly contagious. It has spread extremely rapidly from a single city to the entire country within only about 30 days. Moreover, it has achieved such far-reaching effects even in the face of extreme response measures including the complete shutdown and isolation of whole cities, cancellation of Chinese New Year celebrations, prohibition of attendance at school and work, massive mobilization of health and public health personnel as well as military medical units, and rapid construction of entire hospitals.

In light of this rapid spread, it is fortunate that COVID-19 has been mild for $81 \%$ of patients and has a very low overall case fatality rate of $2.3 \%$. Among the
1,023 deaths, a majority have been $\geq 60$ years of age and/or have had pre-existing, comorbid conditions such as hypertension, cardiovascular disease, and diabetes. Moreover, the case fatality rate is unsurprisingly highest among critical cases at $49 \%$, and no deaths have occurred among those with mild or even severe symptoms (Table 1 ).

A major contribution of our study is a first description of the COVID-19 epidemic curves. We interpret the overall curve (Figure 3A) as having a mixed outbreak pattern - the data appear to indicate a continuous common source pattern of spread in December and then from early January through February 11, 2020, the data appear to have a propagated source pattern. This mixed outbreak time trend is consistent with the working theory that perhaps several zoonotic events occurred at Huanan Seafood Wholesale Market in Wuhan allowed 2019$\mathrm{nCoV}$ to be transmitted from a still-unknown animal into humans and, due to its high mutation and recombination rates, it adapted to become capable of and then increasingly efficient at human-to-human transmission $(3,8)$.

The early days of the outbreak have been reminiscent of SARS and MERS, and indeed, the discovery that the causative agent was a closely-related, never-before-described coronavirus predicted potential for nosocomial transmission and so-called "superspreader" events (8). Unfortunately, 2019-nCoV did indeed infect health workers in China via nosocomial transmission. Here we offer a first description of the 1,716 confirmed cases among health workers. Overall, they also display a likely mixed outbreak pattern - perhaps the data are characterized by a point source curve beginning in late December 2019, which

TABLE 2. Confirmed cases, case severity, and case fatality rates among health workers in different areas of China by time period.

\begin{tabular}{|c|c|c|c|c|c|c|c|c|c|c|c|c|}
\hline \multirow[b]{2}{*}{$\begin{array}{c}\text { Period } \\
\text { (by date of onset) }\end{array}$} & \multicolumn{3}{|c|}{ Wuhan } & \multicolumn{3}{|c|}{ Hubei (outside Wuhan) } & \multicolumn{3}{|c|}{ China (outside Hubei) } & \multicolumn{3}{|c|}{ China (overall) } \\
\hline & $\begin{array}{c}\text { Confirmed } \\
\text { cases, } \\
\mathbf{N} \\
\end{array}$ & $\begin{array}{c}\text { Severe + } \\
\text { critical, } \\
\mathrm{N}(\%)\end{array}$ & $\begin{array}{l}\text { Deaths, N } \\
(\text { CFR* } \%)\end{array}$ & $\begin{array}{l}\text { Confirmed } \\
\text { cases, } \\
\mathrm{N}\end{array}$ & $\begin{array}{c}\text { Severe + } \\
\text { critical, } \\
\mathrm{N}(\%) \\
\end{array}$ & $\begin{array}{l}\text { Deaths, N } \\
(\text { CFR }, \%)\end{array}$ & $\begin{array}{c}\text { Confirmed } \\
\text { cases, } \\
\mathrm{N}\end{array}$ & $\begin{array}{c}\text { Severe + } \\
\text { critical, } \\
\mathrm{N}(\%) \\
\end{array}$ & $\begin{array}{l}\text { Deaths, N } \\
(\text { CFR }, \%)\end{array}$ & $\begin{array}{l}\text { Confirmed } \\
\text { cases, } \\
\mathrm{N}\end{array}$ & $\begin{array}{c}\text { Severe + } \\
\text { critical, } \\
\mathrm{N}(\%)\end{array}$ & $\begin{array}{l}\text { Deaths, N } \\
\text { (CFR, } \%)^{*}\end{array}$ \\
\hline Before Dec 31, 2019 & 0 & 0 & 0 & 0 & 0 & 0 & 0 & 0 & 0 & 0 & 0 & 0 \\
\hline Jan $1-10,2020$ & 18 & $7(38.9)$ & $1(5.6)$ & 1 & $1(100)$ & 0 & 1 & $1(100)$ & 0 & 20 & $9(45.0)$ & $1(5.0)$ \\
\hline Jan 11-20, 2020 & 233 & $52(22.3)$ & $1(0.4)$ & 48 & $8(16.7)$ & 0 & 29 & $1(3.4)$ & 0 & 310 & $61(19.7)$ & $1(0.3)$ \\
\hline Jan 21-31, 2020 & 656 & $110(16.8)$ & 0 & 250 & $29(11.6)$ & $2(0.8)$ & 130 & $10(7.7)$ & 0 & 1,036 & $149(14.4)$ & $2(0.2)$ \\
\hline After Feb 1, 2020 & 173 & $22(12.7)$ & $1(0.6)$ & 95 & $3(3.2)$ & 0 & 54 & $3(5.6)$ & 0 & 322 & $28(8.7)$ & $1(0.3)$ \\
\hline Total & 1,080 & $191(17.7)$ & $3(0.3)$ & 394 & $41(10.4)$ & $2(0.5)$ & 214 & $15(7.0)$ & 0 & 1,688 & $247(14.6)$ & $5(0.3)$ \\
\hline
\end{tabular}

Abreviation: CFR, case-fatality rate.

* CFR presented here was calculated as number of deaths (numerator) divided by total number of confirmed cases in the row (denominator), expressed as a percent. 
was eclipsed by a higher magnitude continuous source curve beginning on January 20, 2020. To date, there is no evidence of a super-spreader event occurring in any of the Chinese health facilities serving COVID-19 patients. However, we do not know whether this is due to the nature of the virus itself or whether these events have been successfully prevented.

It is these authors' sincere hope and intent that this new analysis, on what has become a "public health emergency of international concern," (12) helps to inform health and public health workers preparing for or perhaps already experiencing COVID-19 in their populations. This study provides important insight into several crucial open questions on this epidemic and how to design strategies to effectively control it (3). For instance, the downward trend in the overall epidemic curve suggests that perhaps isolation of whole cities, broadcast of critical information (e.g., promoting hand washing, mask wearing, and care seeking) with high frequency through multiple channels, and mobilization of a multi-sector rapid response teams is helping to curb the epidemic.

China's response is certainly an echo of lessons learned during SARS and is a tribute to the work China and other low- and middle-income countries have been doing, with the much-needed help of international partners, over the past few decades to build infectious disease surveillance systems and public health infrastructure capable of catching outbreaks early and responding swiftly using evidence-based best practices. The 2019-nCoV and other coronaviruses may continue to adapt over time to become more virulent (3), and zoonosis is not going to stop. We must remain vigilant, hone our skills, fund our defenses, and practice our responses, and we must help our neighbors to do the same.

The very large number of cases included in our study was a major strength. Nevertheless, our study did have some important limitations. Firstly, a large proportion of cases included in our analysis (37\%) were not confirmed by nucleic acid testing since this process is slow, labor intensive, and requires specialized equipment and skilled technicians. Yet all 72,314 cases were at least diagnosed clinically and investigated by trained epidemiologists. Secondly, some records did have missing data for a few important variables of interest-Wuhan-related exposure, comorbid conditions, and case severity-which limits our ability to draw conclusions from the data.

In conclusion, the present descriptive, exploratory analysis of the first 72,314 cases of COVID-19 reported through February 11, 2020 offers important new information to the international community on the epidemic in China. In particular, this analysis chronicles the extremely rapid spread of the novel coronavirus despite extreme efforts to contain it. However, important questions remain including identification of the animal reservoir, determination of infectiousness period, identification of transmission routes, and effective treatment and prevention methods including further test development, drug development, and vaccine development (3-4,8-9). As an international community, we must all be responsible partners in surveillance, communication, response, research, and implementation of evidence-based public health and clinical practice. The massive vigorous actions taken by the Chinese government have slowed down the epidemic in China and curbed spread to the rest of the world. Although the epidemic appears to be in decline in the lead up to February 11, 2020, we may yet face more challenges. Huge numbers of people will soon be returning to work and school after the extended New Year holiday. We need to prepare for a possible rebound of the COVID-19 epidemic in the coming weeks and months.

\section{Acknowledgements}

This work was supported by National Science and Technology Foundation of China (2018ZX10201002008-002), and by National Nature Science Foundation of China (NSFC, 71934002). The funder had no role in the design and conduct of the study; collection, management, analysis, and interpretation of the data; preparation, review, or approval of the manuscript; and decision to submit the manuscript for publication.

The team thanks all local health workers for their contributions in providing testing, treatment, and care to COVID-19 patients in China.

Disclaimer: The opinions expressed herein reflect the collective views of the co-authors and do not necessarily represent the official position of the National Center for AIDS/STD Control and Prevention of the Chinese Center for Disease Control and Prevention.

In order to share the results of epidemiological characteristics of COVID-19 domestically and internationally, the Chinese Versionis jointly published on the Chinese Journal of Epidemiology. 
Author Group \& Contributions: The Novel Coronavirus Pneumonia Emergency Response Epidemiology Team includes Zijian Feng, Qun Li, Yanping Zhang", Zunyou Wu, Xiaoping Dong, Huilai Ma, Dapeng Yin, Ke Lyu, Dayan Wang, Lei Zhou, Ruiqi Ren, Chao Li, Yali Wang, Dan Ni, Jing Zhao, Bin Li, Rui Wang, Yan Niu, Xiaohua Wang, Lijie Zhang, Jingfang Sun, Boxi Liu, Zhiqiang Deng, Zhitao Ma, Yang Yang, Hui Liu, Ge Shao, Huan Li, Yuan Liu, Hangjie Zhang, Shuquan Qu, Wei Lou, Dou Shan, Yuehua Hu, Lei Hou, Zhenping Zhao, Jiangmei Liu, Hongyuan Wang, Yuanjie Pang, Yuting Han, Qiuyue Ma, Yujia Ma, Shi Chen, Wei Li, Routong Yang, Zhewu Li, Yingnan Guo, Xinran Liu, Bahabaike Jiangtulu, Zhaoxue Yin, Juan $\mathrm{Xu}$, Shuo Wang, Lin Xiao, Tao Xu, Limin Wang, Xiao Qi, Guoqing Shi, Wenxiao Tu, Xiaomin Shi, Xuemei Su, Zhongjie Li, Huiming Luo, Jiaqi Ma, Jennifer M. McGoogan. All Team members jointly conceptualized the study, analyzed and interpreted the data, wrote and revised the manuscript, and decided to submit for publication.

\# Corresponding author: Yanping Zhang, zhangyp@chinacdc.cn.

Submitted: February 14, 2020; Accepted: February 14, 2020

\section{References}

1. Wuhan Municipal Health Commission. Report of clustering pneumonia of unknown etiology in Wuhan City. Wuhan, China: Wuhan Municipal Health Commission. http://wjw.wuhan.gov.cn/ front/web/showDetail/2019123108989. [2019-12-31]. (In Chinese).

2. World Health Organization. Novel coronavirus - China. Geneva, Switzerland: World Health Organization. https://www.who.int/csr/ don/12-january-2020-novel-coronavirus-china/en/. [2020-01-12].

3. Wang C, Hornby PW, Hayden FG, Gao GF. A novel coronavirus outbreak of global health concern. Lancet. http://dx.doi.org/10. 1016/S0140-6736(20)30185-9. [2020-01-24].

4. Hui DS, Azhar EI, Madani TA, Ntoumi F, Kock R, Dar O, et al. The continuing 2019-nCoV epidemic threat of novel coronaviruses to global health - the latest 2019 novel coronavirus outbreak in Wuhan, China. Int J Infect Dis 2020;91(2020):264 - 6. http://dx.doi.org/10.1016/ j.ijid.2020.01.009.

5. Zhu N, Zhang D, Wang W, Li XW, Yang B, Song JD, et al. A novel coronavirus from patients with pneumonia in China, 2019. N Engl J Med. http://dx.doi.org/10.1056/NEJMoa2001017. [2020-01-24].

6. Chan JFW, Kok KH, Zhu Z, Chu H, To KKW, Yuan SF, et al. Genomic characterization of the 2019 novel human-pathogenic coronavirus isolated from a patient with atypical pneumonia after visiting Wuhan. Emerg Microbs Infect. http://dx.doi.org/10.1080/ 22221751.2020.1719902. [2020-01-24].

7. Tan WJ, Zhao X, Ma XJ, Wang WL, Niu PH, Xu WB, et al. A novel coronavirus genome identified in a cluster of pneumonia cases-Wuhan, China 2019-2020. China CDC Weekly 2020;2(4): 612. http://weekly.chinacdc.cn/en/article/ccdcw/2020/4/61.

8. Paules CI, Marston HD, Fauci AS. Coronavirus infection-more than just the common cold. JAMA. http://dx.doi.org/10.1001/jama.2020. 0757. [2020-01-23].

9. Munster VJ, Koopmans M, van Doremalen N, van Riel D, de Wit E. A novel coronavirus emerging in China - key questions for impact assessment. N Engl J Med. http://dx.doi.org/10.1056/NEJMp2000929. [2020-01-24].

10. Huang C, Wang Y, Li X, Ren L, Zhao J, Hu Y, et al. Clinical features of patients infected with 2019 novel coronavirus in Wuhan, China. Lancet. http://dx.doi.org/10.1016/S0140-6736(20)30183-5. [2020-0124]

11. Chan JFW, Yuan S, Kok KH, To KKW, Chu H, Yang J, et al. A familial cluster of pneumonia associated with the 2019 novel coronavirus indicating person-to-person transmission: a study of a family cluster. Lancet. http://dx.doi.org/10.1016/S0140-6736(20) 30154-9. [2020-01-24].

12. World Health Organization. Statement on the second meeting of the International Health Regulations (2005) Emergency Committee regarding the outbreak of novel coronavirus (2019-nCoV). Geneva, Switzerland: World Health Organization. https://www.who.int/news$\mathrm{room} /$ detail/30-01-2020-statement-on-the-second-meeting-of-theinternational-health-regulations-(2005)-emergency-committee-regar ding-the-outbreak-of-novel-coronavirus-(2019-ncov). [2020-01-30]. 